\title{
BMJ Open Determinants of inter-practice variation in childhood asthma and respiratory infections: cross-sectional study of a national sentinel network
}

Uy Hoang, ${ }^{1}$ Harshana Liyanage, ${ }^{1}$ Rachel Coyle, ${ }^{1}$ Charles Godden, ${ }^{2}$ Simon Jones, ${ }^{1,3}$ Mitch Blair, ${ }^{4}$ Michael Rigby, ${ }^{5}$ Simon de Lusignan ${ }^{1,6}$

To cite: Hoang U, Liyanage $\mathrm{H}$, Coyle R, et al. Determinants of inter-practice variation in childhood asthma and respiratory infections: crosssectional study of a national sentinel network. BMJ Open 2019;9:e024372. doi:10.1136/ bmjopen-2018-024372

\section{- Prepublication history for} this paper is available online. To view these files, please visit the journal online (http://dx.doi. org/10.1136/bmjopen-2018024372).

Received 29 May 2018 Revised 24 September 2018 Accepted 5 December 2018

Check for updates

(C) Author(s) (or their employer(s)) 2019. Re-use permitted under CC BY-NC. No commercial re-use. See rights and permissions. Published by BMJ.

For numbered affiliations see end of article.

Correspondence to

Dr Uy Hoang;

u.hoang@surrey.ac.uk

\section{ABSTRACT}

Objectives Respiratory infections are associated with acute exacerbations of asthma and accompanying morbidity and mortality. In this study we explore interpractice variations in respiratory infections in children with asthma and study the effect of practice-level factors on these variations.

Design Cross-sectional study.

Setting We analysed data from 164 general practices in the Royal College of General PractitionersResearch and Surveillance Centresentinel network in England.

Participants Children $5-12$ years.

Interventions None. In this observational study, we used regression analysis to explore the impact of practice-level determinants on the number of respiratory infections in children with asthma.

Primary and secondary outcome measures We describe the distribution of childhood asthma and the determinants of upper/lower respiratory tract infections in these children.

Results $83.5 \%$ (137/164) practices were in urban locations; the mean number of general practitioners per practice was 7 ; and the mean duration since qualification 19.7 years. We found almost 10 -fold difference in the rate of asthma (1.5-11.8 per 100 children) and 50-fold variation in respiratory infection rates between practices. Larger practices with larger lists of asthmatic children had greater rates of respiratory infections among these children.

Conclusion We showed that structural/environmental variables are consistent predictors of a range of respiratory infections among children with asthma. However, contradictory results between measures of practice clinical care show that a purely structural explanation for variability in respiratory infections is limited. Further research is needed to understand how the practice factors influence individual risk behaviours relevant to respiratory infections.

\section{INTRODUCTION AND AIMS}

Asthma is the most common long-term condition in children and young people in the UK. The British Lung Foundation estimates that there are 1.1 million children in
Strengths and limitations of this study

- This study used a large, nationally representative data set which allowed us to explore inter-practice variations in diagnosis of asthma among children and examine the determinants of a wide range of respiratory tract infections in these children than has previously been published.

- However, our first limitation is that we used routinely collected data from general practice which was not collected for clinical or epidemiological research.

- Second, our enumeration of practice structural variables relied on publicly available data about practices from their own websites, NHS Choices and the medical register held by the General Medical Council.

- Third, our study also lacked information on other structural and individual variables which may be important in the onset of respiratory infections in children with asthma, including exposure to smoking and pollution which may influence the risk of some respiratory infections in children.

- Finally, inference about the individual risk of respiratory infection in children with asthma is not straightforward from the results of our study, thus it is difficult to deduce changes to individual behaviours that may affect infection risk or make conclusions about changes in clinical practice for individual children.

the UK affected by the condition, ${ }^{1}$ although recent evidence suggests that the prevalence of asthma is declining in certain Western countries. ${ }^{2}$

The condition is characterised by reversible airflow limitation experienced as coughing, wheezing, chest tightness and breathlessness, with symptoms often worse in the early morning and at night. ${ }^{3}$

Infections of the airways in children and adults with asthma may be associated with acute disease exacerbations and the development of chronic disease. ${ }^{4}$ In the UK, asthma 
is among the most common reason for urgent admissions to hospital in young people with many admissions resulting from respiratory infections. ${ }^{5}$ Acute exacerbations of asthma are associated with decreased lung growth or accelerated loss of lung function, ${ }^{6}$ thus prevention and rapid treatment of respiratory infections in this group is crucial to acute and long-term management.

Paediatric asthma in the UK is predominantly managed by general practitioners (GPs) in primary care. ${ }^{7}$ Information collected for the National Health Service (NHS) showed widespread geographical variations, including variations at the practice level in the quality of care and outcomes experienced by people with respiratory disease including asthma in England. ${ }^{8-11}$ However, there is little information about how these geographical variations, especially variations at the practice level, affect the occurrence of respiratory infections in children with asthma.

In this study, we describe variations in respiratory infections among children with asthma registered with general practices who are members of the Royal College of General Practitioners Research and Surveillance Centre (RCGP RSC) network, ${ }^{12}$ the English national sentinel system, and study the effect of practice-level factors on these variations.

\section{METHODS}

\section{Subjects and setting}

We used information from the RCGP RSC. It hosts data as a pseudonymised data set from a nationally representative sample of just under 2 million people registered with 164 of the network practices in the UK. ${ }^{12}$ It has been involved in surveillance of influenza and respiratory disease for $>50$ years. Over this period practices have had feedback about their data quality around influenza and respiratory disease. In particular, the differentiation of first or new (incident) from follow-up consultations. Data quality is good for routine primary care. ${ }^{13}$

UK general practice is suitable for this type of study because it has a registration-based system with patients registered with a single practice. Practices have been computerised since the late 1990s, with pay-for-performance introduced in 2004 for chronic disease management including asthma. ${ }^{14}$ Key data are coded, ${ }^{15}$ which includes diagnoses, therapy, test results and other data.

Asthma was defined using an ontological approach. ${ }^{16} 17$ We combined clinician-assigned diagnostic codes relating to five supporting concepts: (1) diagnostic codes (including those referred to in Quality Outcomes Framework code lists); (2) symptoms of asthma (including wheeze, cough, shortness of breath and chest tightness); (3) diagnostic tests (including peak expiratory flow rates and spirometry); (4) therapies codes (including shortacting beta 2 agonists, inhaled corticosteroids, long-acting beta 2 agonists, leukotriene antagonists and oral steroids); and (5) Process of Care codes (codes for attending an asthma clinic or an asthma medication review that imply a diagnosis of asthma). ${ }^{1819}$

The diagnosis of asthma in very young children under 5 years of age can be difficult. ${ }^{20}$ Disease remission from adolescence also occurs in a significant proportion of children with asthma, ${ }^{21}$ thus we restricted our analysis to children with asthma aged between 5 and 12 years of age on 31st December 2016.

\section{Respiratory infection outcomes}

We conducted a cross-sectional study examining the distribution of both lower and upper respiratory tract infections in children with asthma between 1 January and 31 December 2016.

We extracted information on lower respiratory tract infections (LRTI) including acute bronchitis and bronchiolitis, pneumonia and pneumonitis, pleurisy and other LRTIs. Influenza-like-illness (ILI), which affects both upper and lower respiratory tracts, was grouped with LRTI. We extracted information on upper respiratory tract infections (URTIs) including common cold, streptococcal sore throat, scarlatina and other URTI.

\section{General practice-level characteristics}

We collected the following information about the primary care practices within the RCGP RSC sentinel practice network from the individual practice websites, the NHS Choices website ${ }^{22}$ and the General Medical Council (GMC) register ${ }^{23}$ between 1 August and 31 August 2017 including the total number of GPs in the practice, the gender of the GPs in the practice, the average number of years since their medical and specialist general practice qualifications, the average number of qualifications of GPs in the practice and whether the practice was in an urban or rural location.

We collected information about childhood immunisation rates for each practice from the RCGP RSC in 2016, including (1) diphtheria; tetanus; whooping cough (pertussis); polio; Haemophilus influenzae type b $(\mathrm{DTaP} / \mathrm{IPV} / \mathrm{HiB})$ vaccination rates for children under 12 months; (2) pneumococcal conjugate vaccination (PCV) rates for children under 12 months and (3) live-attenuated influenza vaccination rates for children between 2 and 17 years of age.

We collected information about respiratory care for each practice from the National General Practice Profiles published by Public Health England, including the total achievement score in the respiratory disease domain of the chronic disease pay-for-performance scheme $(\mathrm{P} 4 \mathrm{P})$ the Quality and Outcomes Framework (QOF). Between 2015 and 2016 this included recording the percentage of practice patients aged 8 and over with asthma who have had an asthma review within the past 12 months including an assessment of their asthma control using the Royal College of Physicians three questions survey ${ }^{24}$ and those over the age of 14 years with asthma who have had a record of their smoking status in the last 12 months. 
General practice-level characteristics of children with asthma The following information on the population of children with asthma within each general practice was collected including asthma prevalence, average age of children with asthma, percentage of female and percentage of non-white ethnicity.

The severity of the disease in the practice population of children with asthma was recorded from the proportion prescribed 6 or greater number of inhaled bronchodilators (relievers) in the past year and the proportion prescribed 12 or greater number of inhaled preventers in the past year. ${ }^{25}$

\section{Statistical analysis}

Given the skewed distribution of respiratory infection rates in asthmatic children, we examined the effect of practice-level factors on the count of respiratory infections in children with asthma. Poisson, negative binomial and zero-inflated Poisson regression models of childhood respiratory infection counts in asthmatic children were examined. ${ }^{26}$ Model goodness of fit was compared using the $\log$ likelihood ratio test. ${ }^{27}$ All statistical analyses were undertaken using $\mathrm{R}^{28}$

\section{RESULTS}

\section{Characteristics of practices within the RCGP RSC network}

Information from 164 general practices in the RCGP RSC national sentinel practice network was included in the study. $83.5 \%(137 / 164)$ practices were located in an urban location. The mean number of GPs per practice was 7 (median $=7$ ), and the average number of years since GPs had qualified from medical school was 19.7 years (median=19). Each GP within the practice had on average more than two specialist qualifications in addition to their medical qualifications.

The GP practices within the network achieved on average $97.7 \%$ (95\% CI 97.0 to 98.3 ) of the total $\mathrm{P} 4 \mathrm{P} /$ QOF scores overall and $99.2 \%$ (95\% CI 98.7 to 99.7) of the total $\mathrm{P} 4 \mathrm{P} / \mathrm{QOF}$ scores available for care of respiratory patients. This compares with an average total $\mathrm{P} 4 \mathrm{P} /$ QOF score of $95.5 \%$ (95\% CI 95.3 to 95.6 ) and average $\mathrm{P} 4 \mathrm{P} / \mathrm{QOF}$ scores for care of respiratory patients of $97.4 \%$ (95\% CI 97.2 to 97.6) for all practices in England.

\section{Asthmatic children within the RCGP RSC}

In total, 6161 patients with asthma aged 5-12 years were included in the study. The rate of childhood asthma among practices within the network varied almost 10 -fold between 1.5 per 100 children and 11.8 per 100 children with a mean of 4.1 per 100 children registered with the practice having a diagnosis of asthma (see figure 1).

Asthma rates varied by 2.6-fold when practice rates were grouped together into larger sustainability and transformation partnership (STP) areas (see figure 2). These larger geographical units encompass NHS organisations and local councils in England who are working together

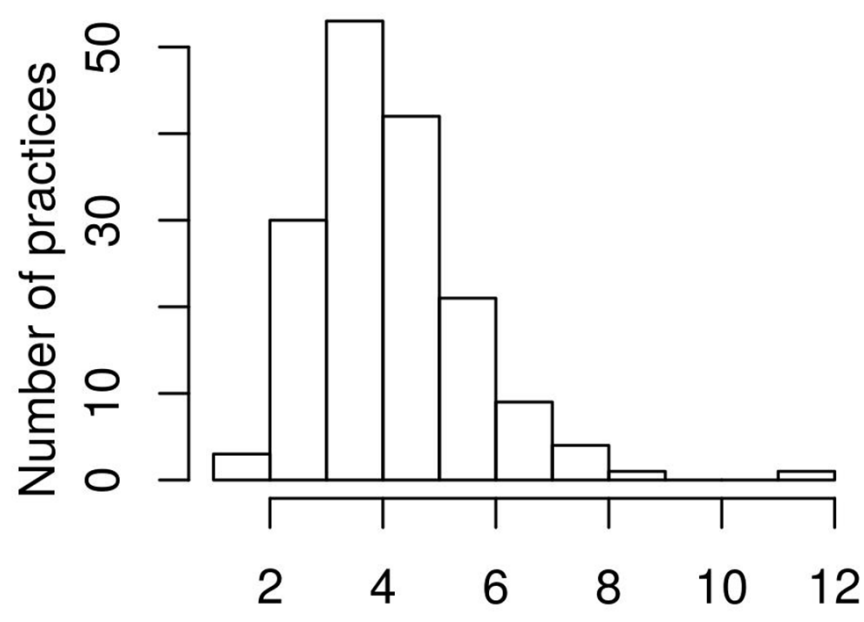

\section{Rate of asthma per 100 children}

Figure 1 Rates of asthma among children aged 5-12 years.

to develop shared care pathways for people with chronic disease. $^{29}$

The severity of asthma among children within the RCGP RSC practices also varied widely as shown by the number that required $>6$ inhaled relievers or 12 inhaled preventers within the past year (see figure 3 ).

\section{Respiratory infection rates in children with asthma}

The rate of infections of the lower respiratory tract in children with asthma varied up to 50 -fold between practices across the network, with rates of ILI showing the widest variation between $0-50$ per 100 children with asthma. Also the rate of infections of the upper respiratory tract in children with asthma almost 20 -fold between practices across the network, with rates of other URTIs showing the widest variation between 3.03 and 56.25 per 100 children with asthma. Table 1 shows the mean respiratory infection rates in children with asthma for practices across the network, the variance and median infection rates.

\section{Determinants of respiratory infection in children with asthma}

We present the negative binomial regression models which produced the most optimal fit for the data. The models showed that the most consistent predictor of both URTIs and LRTIs in children with asthma was the number of children registered with the practice, especially asthmatic children.

The relative risks indicates that as practice list size increased by 50 children there was a $4 \%-9 \%$ greater risk of respiratory infections in their population of asthmatic children.

Equally as the prevalence of children with asthma increased within practices the risk of respiratory infections increased between $19 \%$ and $46 \%$ (see tables 2 and $3)$. 

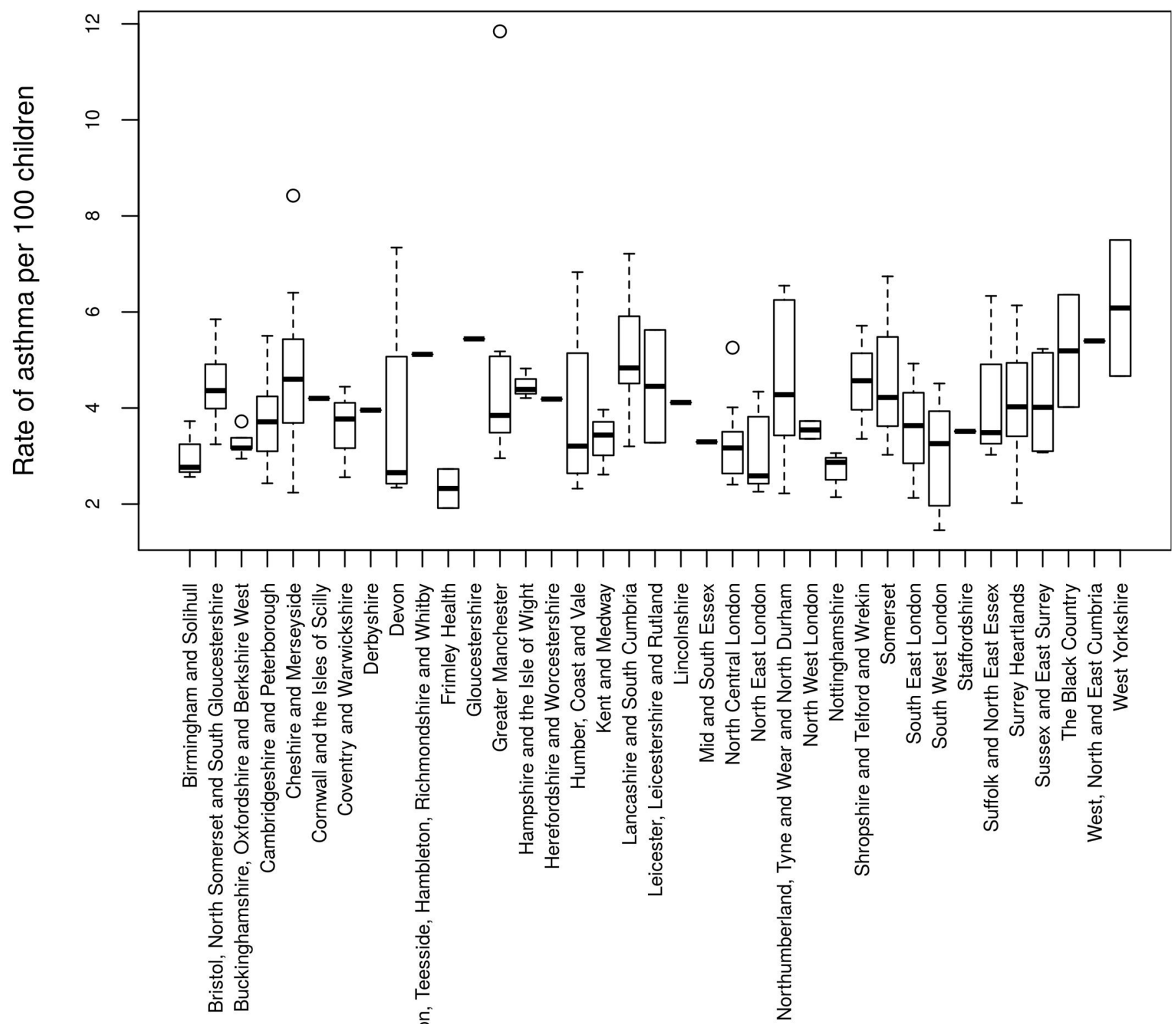

Figure 2 Asthma rates by sustainability and transformation partnership.

\section{DISCUSSION}

\section{Summary of principal findings}

Using data from a national sentinel network, we found wide variation in the prevalence of paediatric asthma in primary care, with almost 10 -fold difference in the rate between practices and 2.6-fold variation between STP areas. We also found widespread variations in the respiratory infections among children with asthma, with an almost 50 -fold difference in the rate between practices. Larger practices with larger lists of asthmatic children tended to have greater rates of respiratory infections among these children.

\section{Strengths and weaknesses of the study}

There are a number of limitations to our study related to the use of routinely collected data from general practice, including the quality of data recording in primary care records. However, our sentinel network has been involved in monitoring ILI and respiratory infections for over 50 years and has had regular feedback on data quality. ${ }^{1213}$ Our previous work has shown that an ontological approach 


\section{Prescription for inhaled relievers $>6$}

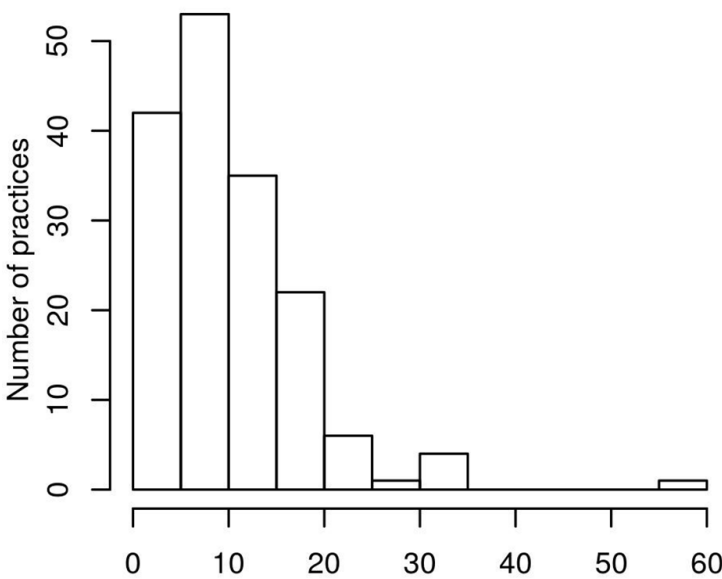

Prescription rate per 100 children with asthma
Prescription for inhaled preventers $>12$

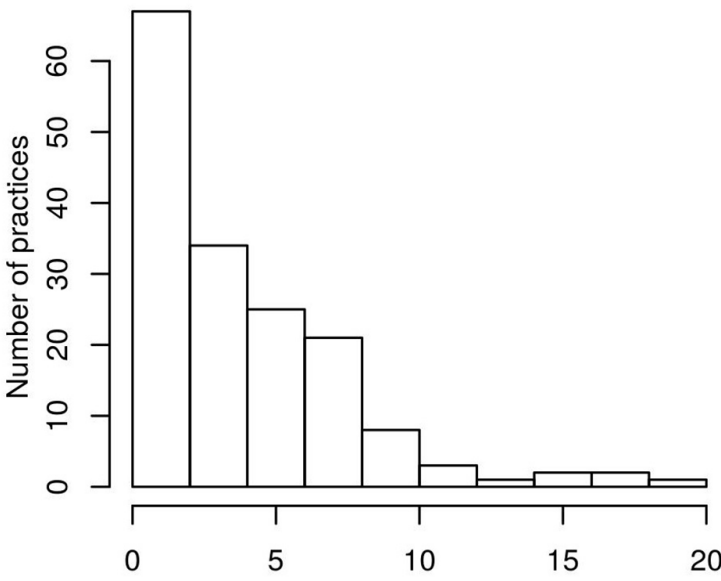

Prescription rate per 100 children with asthma

Figure 3 Severity of asthma among children aged 5-12 years in Royal College of General Practitioners Research and Surveillance Centre practices as indicated by rates of prescribing for $>6$ inhaled relievers or $>12$ inhaled preventers per year.

to asthma case definition combining clinician-assigned codes relating to five supporting concepts of asthma, namely diagnosis, symptoms, diagnostic tests, therapies and processes of care, accurately estimates the prevalence of asthma in a primary care data set compared with published data. However, the accuracy of our ontological definition of asthma has not been specifically tested in populations of children and young people.

An additional limitation relates to the enumeration of practice structural variables as this relied on the extraction of publicly available data about practices from their own websites, NHS Choices and the medical register held by the GMC. The quality of this information, especially it completeness, consistency, timeliness and accuracy, has not been validated in published data, and given the number of practices involved in this study we did not attempt to validate the accuracy with individual practice staff.

Our study also lacked information on other structural and individual variables which may be important in the onset of respiratory infections in children with asthma, including exposure to smoking and pollution which may influence the risk of some respiratory infections in children.

Finally, inference about the individual risk of respiratory infection in children with asthma is not straightforward from the results of our study, thus it is difficult to deduce changes to individual behaviours that may affect infection risk or make conclusions about changes in clinical practice for individual children.

Table 1 Variation between practices in Royal College of General Practitioners Research and Surveillance Centre sentinel network in rates of respiratory infections among children with asthma

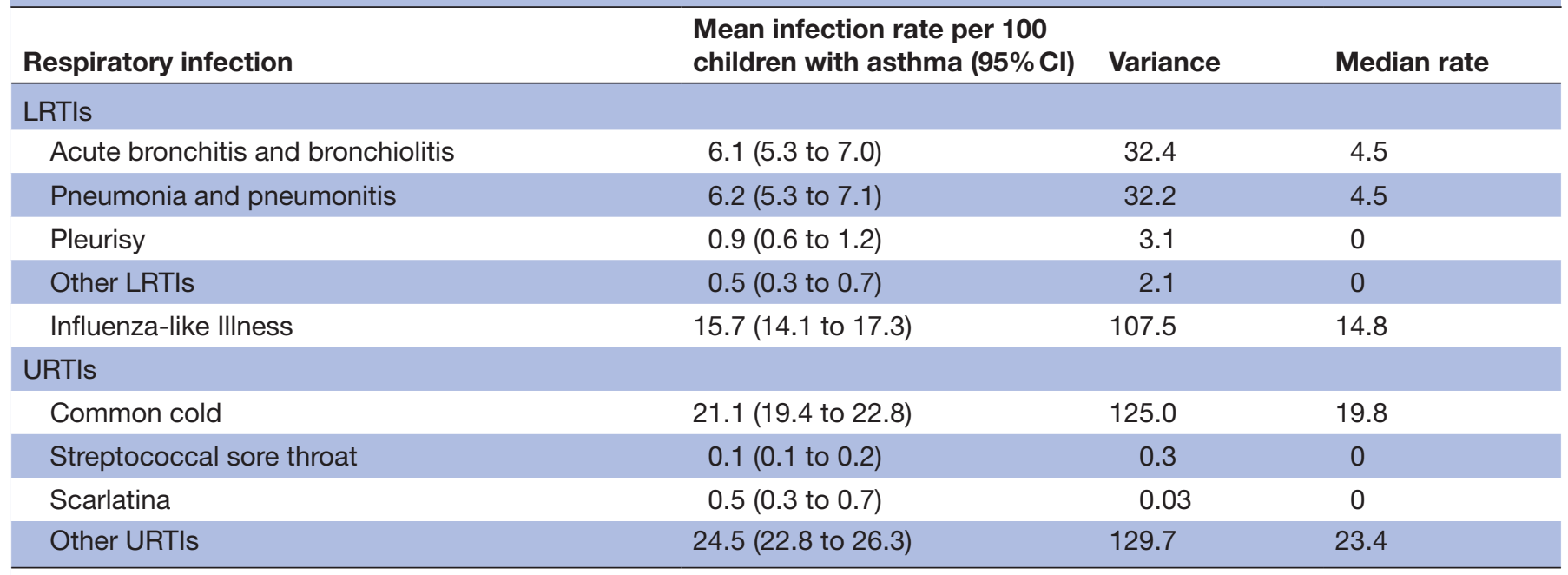

LRTIs, lower respiratory tract infections; URTIs, upper respiratory tract infections. 


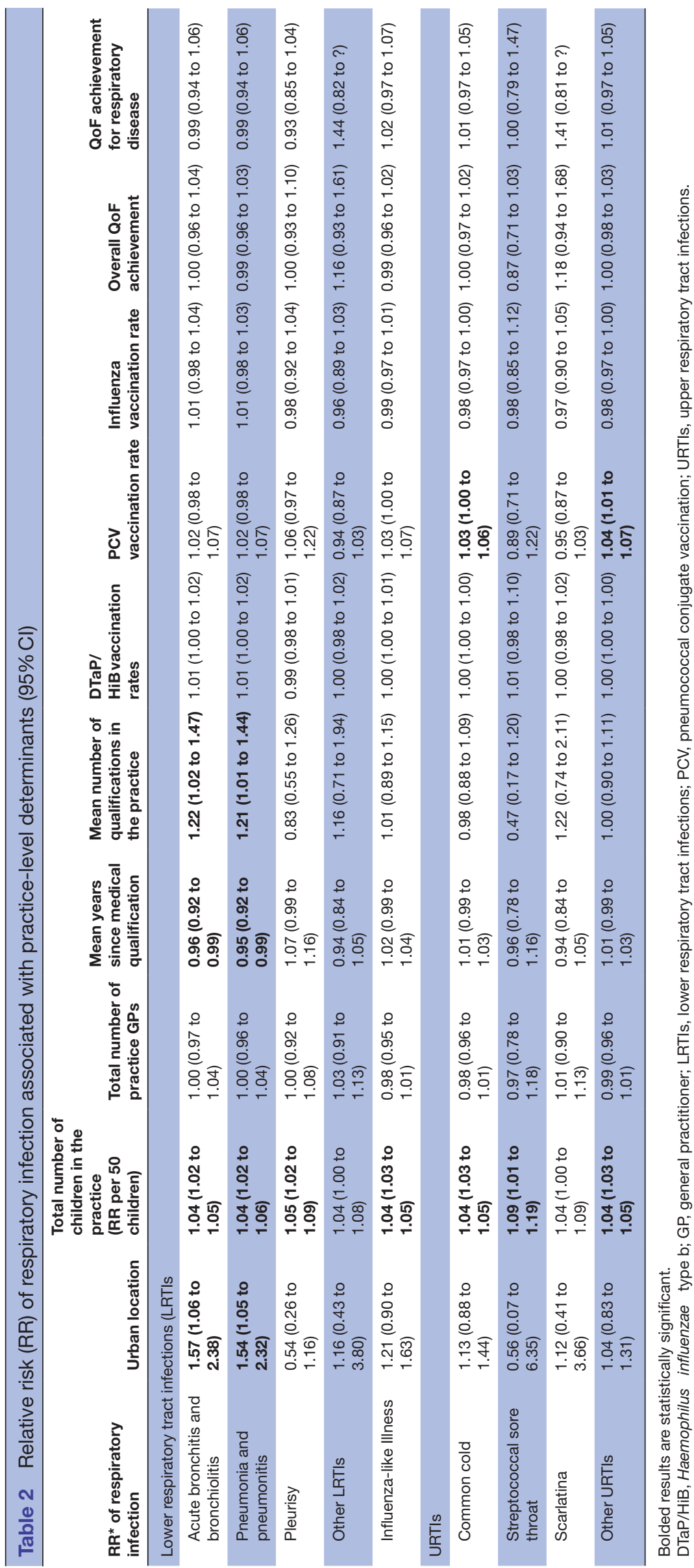




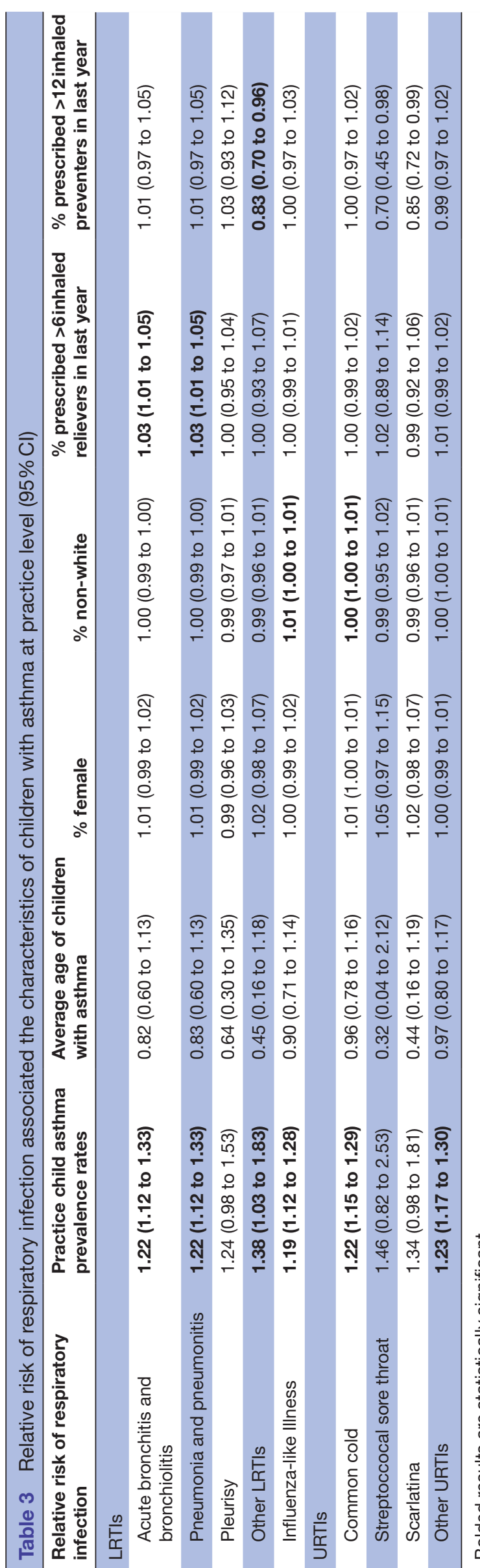

However, our study has a number of strengths including the fact that we used a large, nationally representative data $\operatorname{set}^{12}$ which allowed us to examine a wide number of URTIs and LRTIs in this group of children compared with previously published research.

\section{Strengths and weaknesses in relation to other studies, discussing important differences in results}

Our study shows widespread inter-practice variations in asthma prevalence and variations in respiratory infections. These variations could indicate variations in the recording of asthma and respiratory infections in primary care, although we have previously shown that the prevalence of asthma in our database is in line with previously published data which suggest that recording of cases in the RCGP RSC network is in line with other general practices.

While our study looked at the effect of variations in clinical care provided for children with asthma, the indicators of the quality of clinical care provided for children with asthma used in this study, namely the $\mathrm{P} 4 \mathrm{P} /$ QOF indicators, do not specifically include measures of respiratory care in children and showed little variability. In fact practice measures of clinical care either showed no or contradictory results such as the practice vaccination rates of PCV and influenza which showed increase or decrease in respiratory infections, respectively. These practice measures of clinical care may only be partially capturing changes in individual behaviours that change risk of respiratory infections and there is a need for a better understanding of how structural/environmental factors influence individual risk and vice versa.

\section{Meaning of the study: possible explanations and implications for clinicians and policymakers}

Previous studies have shown that asthma is familial and that the genetic makeup of stable populations suggests that the probable cause of variations in asthma prevalence must lie in the environment. ${ }^{30}$

Various environmental exposures have been posited as causally related to asthma prevalence including air pollution, airborne allergens, house dust mite and smoking. ${ }^{31}$ There is also some evidence that these exposures may be causally related to respiratory infections. ${ }^{32}$

An alternative explanation for our findings may be the result of population-level variations in genetic susceptibility causing both asthma and asthma-related infections. $^{31}$

In such a scenario, temporal and geographical variations in asthma rates would closely mirror respiratory infection rates with little influence from changes in environmental exposures over time or geography.

Lastly our findings suggest the need for the improved monitoring of respiratory infections in children with asthma, especially those from large urban practices who are at increased risk of infections that could result in acute exacerbations or chronic disease. 


\section{Unanswered questions and future research}

The findings from our study raise a number of important questions for further research. First, are the variations in asthma rates and respiratory infection rates between practices also seen over time and are structural factors equally important in determining temporal variations in these rates?

Second, what other structural and environmental variables or individual exposures are important determinants of variations in asthma prevalence and respiratory infection rates in this population? For example, are there important genetic variations between populations that could result in these variations in asthma and asthma-related respiratory infections? How do the structural/ environmental factors influence individual children with asthma or the clinicians caring for them to change the risk of infection and vice versa?

\section{Author affiliations}

${ }^{1}$ Department of Clinical and Experimental Medicine, University of Surrey, Guildford, UK

${ }^{2}$ St Mary's Hospital, Isle of Wight, UK

${ }^{3}$ Division of Healthcare Delivery Science/ Center for Healthcare Innovation and Delivery Science (CHIDS), Department of Population Health, New York University, Langone Medical Centre, New York, USA

${ }^{4}$ Department of Paediatrics and Child Health, Northwick Park Hospital, Harrow, UK ${ }^{5}$ Section of Paediatrics, School of Medicine, Imperial College London, St. Mary's Hospital, London, UK

${ }^{6}$ Research and Surveillance Centre, Royal College of General Practitioners, London, UK

Contributors UH, SdL, MB, SJ and HL were responsible for the conception or design of the work. $\mathrm{UH}$ and $\mathrm{HL}$ were responsible for data analysis and writing the initial draft manuscript. UH, HL, RC, CG, SJ, MB, MR and SdL were responsible for interpretation of data, revising the manuscript critically for important intellectual content and final approval of the version to be published.

Funding This study was based on work as part of the Models of Child Health Appraised (MOCHA) project. This project has received funding from the European Union's Horizon 2020 research and innovation programme under the Grant Agreement №. 634201.

Disclaimer The funders did not have any role in study design, analysis or interpretation of data, in the writing of the report or in the decision to submit the article for publication.

Competing interests None declared.

Patient consent Not required.

Ethics approval All data used in this study had been anonymised at the point of data extraction. No clinically identifiable information was used. The study received ethical approval from the South West Central Bristol Research Ethics Committee (REC reference number: 17/SW/0137, approval granted 16 June 2017).

Provenance and peer review Not commissioned; externally peer reviewed.

Data sharing statement Additional data are available by emailing Dr Uy Hoang, u. hoang@surrey.ac.uk.

Open access This is an open access article distributed in accordance with the Creative Commons Attribution Non Commercial (CC BY-NC 4.0) license, which permits others to distribute, remix, adapt, build upon this work non-commercially, and license their derivative works on different terms, provided the original work is properly cited, appropriate credit is given, any changes made indicated, and the use is non-commercial. See: http://creativecommons.org/licenses/by-nc/4.0/.

\section{REFERENCES}

1. Asthma statistics: British Lung Foundation. https://statistics.blf.org. uk/asthma

2. Anderson HR, Gupta R, Strachan DP, et al. 50 years of asthma: UK trends from 1955 to 2004. Thorax 2007;62:85-90.
3. Martinez FD, Vercelli D. Asthma. The Lancet 2013;382:1360-72.

4. Pelaia G, Vatrella A, Gallelli L, et al. Respiratory infections and asthma. Respir Med 2006;100:775-84.

5. Saxena S, Bottle A, Gilbert R, et al. Increasing short-stay unplanned hospital admissions among children in England; time trends analysis '97-'06. PLoS One 2009; 4:e7484.

6. Ahanchian $\mathrm{H}$, Jones $\mathrm{CM}$, Chen YS, et al. Respiratory viral infections in children with asthma: do they matter and can we prevent them? BMC Pediatr 2012;12:147.

7. Abdelhamid AS, Maisey S, Steel N. Predictors of the quality of care for asthma in general practice: an observational study. Fam Pract 2010;27:186-91.

8. Garbutt JM, Yan Y, Strunk RC. Practice Variation in Management of Childhood Asthma Is Associated with Outcome Differences. J Allergy Clin Immunol Pract 2016:4:474-80.

9. Finkelstein JA, Lozano P, Fuhlbrigge AL, et al. Practice-level effects of interventions to improve asthma care in primary care settings: the pediatric asthma care patient outcomes research team. Health Serv Res 2005;40(6 Pt 1):1737-57.

10. Chamberlain JM, Teach SJ, Hayes KL, et al. Practice pattern variation in the care of children with acute asthma. Acad Emerg Med 2016;23:166-70.

11. NHS Atlas of Variation in Healthcare for People with Respiratory Disease. 2012.

12. Correa A, Hinton W, McGovern A, et al. Royal College of General Practitioners Research and Surveillance Centre (RCGP RSC) sentinel network: a cohort profile. BMJ Open 2016;6:e011092.

13. de Lusignan S, Correa A, Smith GE, et al. RCGP Research and Surveillance Centre: 50 years' surveillance of influenza, infections, and respiratory conditions. Br J Gen Pract 2017;67:440-1.

14. de Lusignan $\mathrm{S}$, van Weel $\mathrm{C}$. The use of routinely collected computer data for research in primary care: opportunities and challenges. Fam Pract 2006;23:253-63.

15. de Lusignan S. Codes, classifications, terminologies and nomenclatures: definition, development and application in practice. Inform Prim Care 2005:13:65-9.

16. de Lusignan S, Shinneman S, Yonova I, et al. An ontology to improve transparency in case definition and increase case finding of infectious intestinal disease: database study in english general practice. JMIR Med Inform 2017;5:e34.

17. Liaw ST, Rahimi A, Ray P, et al. Towards an ontology for data quality in integrated chronic disease management: a realist review of the literature. Int J Med Inform 2013;82:10-24.

18. McNulty $\mathrm{R}$, Liyanage $\mathrm{H}$, Arrowsmith $\mathrm{B}$, et al. Time to present the ontological basis of disease prevalence: Asthma as an exemplar, in: Health-exploring complexity: an interdisciplinary systems approach HEC 2016: 28 August-2 September 2016, Munich, Germany. Eur J Epidemiol 2016;131:1-239.

19. McNulty R, Liyanage $\mathrm{H}$, Arrowsmith $\mathrm{B}$, et al. Asthma Ontology: National Center for Biomedical Ontology BioPortal. 2017 http:// bioportal.bioontology.org/ontologies/AO/

20. Pedersen SE, Hurd SS, Lemanske RF, et al. Global strategy for the diagnosis and management of asthma in children 5 years and younger. Pediatr Pulmonol 2011;46:1-17.

21. Bronnimann S, Burrows B. A prospective study of the natural history of asthma. Remission and relapse rates. Chest 1986;90:480-4.

22. NHS Choices. https://www.nhs.uk/pages/home.aspx

23. General Medical Council. List of registered medical practitioners. http://www.gmc-uk.org/doctors/register/LRMP.asp

24. Steven K, Neville RG, Hoskins G, et al. The RCP's 'Three Key Questions' for asthma: review of practical use. Br J Community Nurs 2002;7:300-3.

25. Hoskins G, Williams B, Jackson C, et al. Assessing asthma control in UK primary care: use of routinely collected prospective observational consultation data to determine appropriateness of a variety of control assessment models. BMC Fam Pract 2011;12:105.

26. Jones A, Rice N, d'Uva T, et al. Models for count data. Applied Health Economics. London: Routledge, 2013:295-341.

27. Wilks SS. The large-sample distribution of the likelihood ratio for testing composite hypotheses. Ann Math Statist 1938;9:60-2.

28. RStudio. RStudio, new open-source IDE for R. https://blog.rstudio. com/2011/02/28/rstudio-new-open-source-ide-for-r/

29. Delivering the forward view: NHS planning guidance 2016/172020/21. London: NHS England, 2015.

30. Cookson WO, Moffatt MF. Asthma: an epidemic in the absence of infection? Science 1997;275:41-2.

31. Seaton A, Godden DJ, Brown K. Increase in asthma: a more toxic environment or a more susceptible population? Thorax 1994;49:171-4.

32. Rylander R, Mégevand $Y$. Environmental risk factors for respiratory infections. Arch Environ Health 2000:55:300-3. 